\title{
Modelling and Assessment of Survival Probability of Shock Model with Two Kinds of Shocks
}

\author{
Surekha B. Munoli, Suhas \\ Department of Statistics, Karnatak University, Dharwad, India \\ Email: sbmunoli@yahoo.co.in, suhasbhat2@gmail.com
}

How to cite this paper: Munoli, S.B. and Suhas (2019) Modelling and Assessment of Survival Probability of Shock Model with Two Kinds of Shocks. Open Journal of Statistics, 9, 484-493.

https://doi.org/10.4236/ojs.2019.94033

Received: July 18, 2019

Accepted: August 10, 2019

Published: August 13, 2019

Copyright $\odot 2019$ by author(s) and Scientific Research Publishing Inc. This work is licensed under the Creative Commons Attribution International License (CC BY 4.0).

http://creativecommons.org/licenses/by/4.0/

\section{(c) (i) Open Access}

\begin{abstract}
The study aims at modelling and assessment of survival probability of a component experiencing two kinds of shocks namely, damage shock and fatal shock. Shocks are occurring randomly in time as events of a Poisson Process. The two cases of fixed/random threshold of components are studied. Survival probabilities of proposed models are derived. Maximum likelihood estimators (MLEs) of survival probabilities are obtained using the data from life testing experiments. Fisher information and asymptotic distribution of MLEs of parameters are obtained when a constant threshold is considered. Computation and comparison of estimators of two cases (constant threshold and random threshold) are made through simulation studies. The study recommends the consideration of threshold as a random variable.
\end{abstract}

\section{Keywords}

Damages, Life Testing Experiment, Newton-Raphson Method, Poisson Process, Shock Models, Survival Probability

\section{Introduction}

Study of reliability can be broadly classified into two major aspects i.e. reliability modelling and reliability assessments. Modelling reliability aims at development of reliability model under certain assumptions. Here one discusses about configuration of associated components, methods of reliability enhancement, the conditions under which the system functions at its best, etc. Here the point of interest is "system survival" and compensatory measures to increase system survival.

On the counter part, reliability assessment involves processes such as identifi- 
cation of various modes of system failure, accordingly defining life testing experiments, assigning parameter values to life distributions, repair distributions, threshold distributions and inferential procedures to evaluate the modelled reliability. To assess reliability, one needs data pertaining to system functioning or failure and is obtained through life testing experiments.

Shock models have been studied by several authors. Here are a few references of contributions towards these models. Esary et al. (1973) studied the life distribution properties of a device subjected to a sequence of shocks occurring randomly in time according to a homogeneous Poisson process [1]. Abdel-Hameed and Proschan (1973) extended the results of [1] by assuming shocks that occur according to a nonhomogeneous Poisson process [2]. Barlow and Proschan (1975) discussed different varieties of general and cumulative shock models [3]. Abdel-Hameed and Proschan (1975) and Klefsjo (1981) considered general case in which shocks occur according to a nonstationary pure birth process [4] [5]. Ross (1981) presented a generalized Poisson shock model using increasing failure rate average (IFRA) stochastic processes [6]. Posner and Zuckerman (1986) proposed a replacement model for a semi-Markov shock model with additive damage [7]. Anderson (1987) considered a general shock model in which the time intervals between shocks have infinite expectations [8]. Savits (1988) studied nonhomogeneous Poisson shock processes and the effect of different mean functions for the shock processes on the distributional properties of the joint component lifetimes [9]. Gut (1990) considered theory for stopped two-dimensional random walks to describe cumulative shock models [10]. Skoulakis (2000) studied system reliability subjected to shocks generated by renewal point process [11]. Mallor and Santos (2003) introduced a new shock model by considering the magnitude of the shock and interarrival time between shocks, which generalizes some of the classical shock models [12]. Munoli and Bhat (2011) derived the reliability function of non-accumulating damage shock model with successive shocks causing greater damage. They obtained Maximum Likelihood and Bayes estimators of reliability functions using the data from a type II censored sample without replacement life testing experiment [13]. Lindskog and McNeil (2003) studied Poisson shock models in the context of insurance loss modelling and credit risk modelling [14].

In most of the works cited above, it is observed that shocks are of one type, causing damage to the component (damage shock). But there are some catastrophic shocks whose impact is very high and the component fails at the occurrence of such shocks (fatal shock). The present study endeavours to model and assess survival probability of a component experiencing two kinds of shocks namely, damage shock and fatal shock. The two cases of fixed/random threshold of components are considered. Section 2 deals with the model description and discussions on real life examples. Assessment of Survival function is explained in Section 3. Section 4 deals with validation of derived results through simulation studies. The case of threshold being a random variable is considered in Section 5. Comparison of estimators obtained in two cases of fixed and random threshold 
is outlined in Section 6. Results and findings are also discussed in the same Section.

\section{Modelling Survival Probability}

A component or system is experiencing shocks occurring randomly in time as events of a Poisson process with intensity $\lambda, \lambda>0$. Shocks are of two types. One is fatal shock, which causes failure of the system or component. Another is damage shock, which causes some amount of damage to the component. Damages are non-accumulating. Damages $(X)$ are assumed to be independent and identical exponential random variables with parameter $\theta, \theta>0$. The system or component fails either due to experiencing a fatal shock or whenever the amount of damage due to a damage shock exceeds its threshold $u$. If the damage does not exceed its threshold, the component functions as good as new one. The probability of occurrence of fatal shock is $(1-p)$ and that of damage shock is $p$.

Let us consider threshold of the component as a known constant. The survival probability of the component at time $t$ is given by:

$$
S_{1}(t)=\sum_{k=0}^{\infty} \frac{\mathrm{e}^{(-p \lambda t)}(p \lambda t)^{k}}{k !}\left(1-\mathrm{e}^{-u \theta}\right)^{k} \mathrm{e}^{-(1-p) \lambda t}
$$

The first part of above expression under summation represents the probability that the component experiences $k$ number of damage shocks during $(0, t)$, second part is the probability that the damages due to all $k$ shocks are less than its threshold $u$. The third component is the probability that during $(0, t)$ the component do not experience a fatal shock. During $(0, t)$, the component may experience 0 shock or 1 shock or 2 shocks and so on ... and hence the summation with $k=0,1,2, \cdots$.

Taking the terms independent of $k$ outside the summation and further simplification leads to

$$
S_{1}(t)=\mathrm{e}^{-\lambda t\left[1-p\left(1-\mathrm{e}^{-u \theta}\right)\right]}
$$

$S_{1}(0)=1, S_{1}(\infty)=0, S_{1}(t) \uparrow t$, implying that the survival probability of the component is non-increasing function of time $t$.

The proposed model can be used to describe following real life examples:

Example 1: Heart disease is the leading cause of death for both men and women. Heart is a muscular organ. Most common heart diseases are heart attack and cardiac arrest. But heart attacks don't always kill instantly. Heart attacks occur when blood flow to the heart muscle is temporarily blocked, starving the muscle tissue of oxygen which causes scarring and damage to heart muscle (damage shock with amount of damage tolerable and the person survives with this heart attack). For a heart attack to quickly lead to death, the damage due to the heart needs to be great enough to cause the heart to beat irregularly and eventually stop entirely (damage due to a damage shock exceeding threshold). On the contrary, cardiac arrest occurs suddenly and often without warning. Cardiac arrest is ab- 
rupt loss of heart function, breathing and consciousness. It results from an electric disturbance in the heart that disrupts its pumping action, stopping blood flow to different organs and can lead to death (fatal shock). In this scenario, one will be interested to study the probability that the patient survives or fails with heart attack (damage shock) or with what probability the patient succumbs to cardiac arrest (fatal shock). By studying this, physicians, nutritionist can suggest remedial measures for: avoiding heart attack; if not avoidable, reducing the amount of damage and to make the cardiac arrest (fatal shock) a null event.

Example 2: Credit-scoring systems aid the decision of whether to grant credit to an applicant or not. Traditionally, this is done by estimating the probability that an applicant will default. In recent years the aim has been changing towards choosing the customers of higher profit. In this case it is important to know that when a customer will default (fatal shock). It is possible that if the time to default is long, the acquired interest will compensate or even exceed losses resulting from default. Another factor that affects profitability is the case in which customers close their account early or pay off the loan early by switching to another lender. Depending on when the actual repayment occurred, the lender will lose a proportion of the interest on the loan [15]. One can consider the number of times partial repayments made as damage shocks and the amount repaid is as damage caused due to damage shock. On the other hand, if a customer pays off the loan early then it will be considered as amount of damage due to a damage shock exceeding the threshold (outstanding loan amount). In this case, one will be interested to investigate the probability that a customer will default or the probability that a customer pays off the loan early.

\section{Assessment of the Survival Probability}

Suppose $r$ components each with threshold $u$ having life distribution $H(t)=1-S_{1}(t)$ are subjected to life testing experiment and the experiment is conducted until all of them fail. Out of $r$ components, let $r_{1}$ components fail due to damage shock i.e. damage exceeding the threshold $u$ and $r_{2}\left(=r-r_{1}\right)$ components fail due to occurrence of fatal shock. It is assumed that damage due to fatal shock is not observable. Let the $i^{\text {th }}$ component fail at $n_{i}^{\text {th }}$ shock, $i=1,2, \cdots, r$. Let $X_{i j}, i=1,2, \cdots, r ; j=1,2, \cdots, n_{i}$ be a random variable (r.v.) representing the amount of damage due to $j^{\text {th }}$ damage shock to $i^{\text {th }}$ component. $X_{i j}$ 's are assumed to be independent exponential random variables with parameter $\theta, \theta>0$.

Whenever a component fails due to a damage shock, it is assumed that the damage due to a shock at which the component fails is not observable but is known to exceed the component's threshold $u$. Let $t_{i j}$ be the time epoch at which $j^{\text {th }}$ shock has occurred to $i^{\text {th }}$ component, $j=1,2, \cdots, n_{i} ; i=1,2, \cdots, r$. The inter arrival times $\left(t_{i j}-t_{i j-1}\right)$ are exponential random variables with parameter $p \lambda$. For $r_{1}$ components that fail due to damage exceeding threshold, the joint distribution of r.v.s $n_{i}, t_{i 1}, t_{i 2}, \cdots, t_{i n_{i}}, X_{i 1}, \cdots, X_{i n_{i}-1}$ is 


$$
\prod_{i=1}^{n}(p \lambda)^{n_{i}} \mathrm{e}^{-p \lambda t_{n_{i}}} \theta^{n_{i}-1} \mathrm{e}^{-\theta \sum_{j=1}^{n_{i}-1} x_{i j}} \mathrm{e}^{-u \theta}
$$

Similarly, for $r_{2}$ components that fail due to fatal shock at $n_{i}^{\text {th }}$ shock, the joint distribution of r.v.'s $n_{i}, t_{i 1}, t_{i 2}, \cdots, t_{i n_{i}}, X_{i 1}, \cdots, X_{i n_{i}-1}$ is

$$
\prod_{i=1}^{n_{2}}(p \lambda)^{n_{i}-1} \mathrm{e}^{-p \lambda t_{n_{i}-1}} \theta^{n_{i}-1} \mathrm{e}^{-\theta \sum_{j=1}^{n_{i}-1} x_{i j}}(1-p) \lambda \mathrm{e}^{-(1-p) \lambda} \mathrm{e}^{\left(t_{n_{i}}-t_{n_{i}-1}\right)}
$$

Combining the two cases, the joint distribution $L_{1}$ of all the involved r.v.'s is given by

$$
L_{1}=p^{n \cdot-r_{2}} \lambda^{n} \cdot(1-p)^{r_{2}} \theta^{n \cdot-r} \mathrm{e}^{-p \lambda t} \cdot \mathrm{e}^{-\lambda t^{\prime}} \cdot \mathrm{e}^{-\theta\left(x_{.}+\eta_{1} u\right)}
$$

where,

$$
\begin{gathered}
n=n_{1}+n_{2}, t^{\prime}=\sum_{i=1}^{r_{2}}\left(t_{n_{i}}-t_{n_{i}-1}\right), \\
x_{. .}=\sum_{i=1}^{r} \sum_{j=1}^{n_{i}-1} x_{i j}, t_{. .}=\sum_{i=1}^{n} t_{n_{i}}+2 \sum_{i=1}^{r_{2}} t_{n_{i}-1}-\sum_{i=1}^{r_{2}} t_{n_{i}} .
\end{gathered}
$$

Taking logarithm of sample likelihood $\left(L_{1}\right)$, equating partial derivatives of $\log \left(L_{1}\right)$ with respect to $\theta, p$ and $\lambda$ to zero, we get maximum likelihood estimators of $\theta, p$ and $\lambda$ as

$$
\left.\begin{array}{l}
\hat{\theta}=\frac{n-r}{x_{.}+r_{1} u} \\
\hat{p}=\frac{t^{\prime}\left(n-r_{2}\right)}{t^{\prime} n+t r_{2}} \\
\hat{\lambda}=\frac{n t^{\prime}+r_{2} t}{t^{\prime}\left(t . .+t^{\prime}\right)}
\end{array}\right\}
$$

MLE $\hat{S}_{1}(t)$ of survival function $S_{1}(t)$ is obtained using the MLEs of parameters [16] in expression (2).

Asymptotic Distribution: To obtain the asymptotic distribution of $\hat{\theta}, \hat{p}, \hat{\lambda}$, let us denote the Fisher Information Matrix of $\theta, p, \lambda$ as $I(\theta, p, \lambda)$

$$
I(\theta, p, \lambda)=\left[\begin{array}{ccc}
\frac{n-r}{\theta^{2}} & 0 & 0 \\
0 & b & E\left(t_{. .}\right) \\
0 & E\left(t_{. .}\right) & \frac{n}{\lambda^{2}}
\end{array}\right]
$$

where,

$$
\begin{gathered}
E(t . .)=\sum_{i=1}^{n_{1}} \frac{n_{i}}{p \lambda}+2 \sum_{i=1}^{r_{2}} \frac{n_{i}-1}{p \lambda}-\sum_{i=1}^{r_{2}}\left(\frac{n_{i}-1}{p \lambda}+\frac{1}{(1-p) \lambda}\right), \\
b=\frac{n-r}{p^{2}}+\frac{r_{2}}{(1-p)^{2}}
\end{gathered}
$$

From the asymptotic properties of MLE under regularity conditions and multivariate central limit theorem we have,

$$
[(\theta-\hat{\theta}),(p-\hat{p}),(\lambda-\hat{\lambda})] \rightarrow N_{3}\left(0, I^{-1}\right)
$$


$I^{-1}$ is inverse of Fisher Information Matrix “ $I(\theta, p, \lambda)$ ” and it is given by

$$
I^{-1}=\left[\begin{array}{ccc}
\frac{\theta^{2}}{n-r} & 0 & 0 \\
0 & \frac{n .}{b n-\left(E\left(t_{.}\right) \lambda\right)^{2}} & \frac{-E\left(t_{. .}\right)}{\left(1 / \lambda^{2}\right)\left[b n-\left(E\left(t_{.}\right) \lambda\right)^{2}\right]} \\
0 & \frac{-E\left(t_{.}\right)}{\left(1 / \lambda^{2}\right)\left[b n-\left(E\left(t_{.}\right) \lambda\right)^{2}\right]} & \frac{b}{\left(1 / \lambda^{2}\right)\left[b n .-\left(E\left(t_{.}\right) \lambda\right)^{2}\right]}
\end{array}\right]
$$

\section{Simulation Study}

Validation of proposed model and computation of estimators for different combinations of parameter values is done using Monte-Carlo Simulation. For the $i^{\text {th }}$ component, the random variables $t_{i 1}, t_{i 2}, \cdots, t_{i n_{i}}, X_{i 1}, \cdots, X_{i n_{i}-1}$ are generated as follows:

Step 1: A random number $V_{i}$ is generated from $U(0,1)$. If $0<V_{i}<p=p_{0}$, then it is considered that component has failed due to a damage shock.

Step 2: Initialize $n_{i}=0$; for $\lambda=\lambda_{0}, \theta=\theta_{0}$ the exponential random variable $X_{i 1}$ with parameter $\theta_{0}$ is generated and the value of $n_{i}$ is incremented by one. The process of generation of exponential random variables and incrementation of $n_{i}$ is carried out until $X_{i n_{i}}$ is greater than $u$.

Step 3: $n_{i}$ number of inter arrival times having exponential distribution with parameter $p_{0} \lambda_{0}$ are generated. Addition of these inter arrival times results in $t_{i n_{i}}$. Thus we get $n_{i}, X_{i 1}, \cdots, X_{i n_{i}-1}, t_{n_{i}}$.

Step 4: If $V_{i} \geq p_{0}$, it is considered that the component failure is due to a fatal shock. The process of generation of $n_{i}, X_{i 1}, \cdots, X_{i n_{i}-1}$ is same as Step 2. $n_{i}-1$ number of inter-arrival times having exponential distribution with parameter $p_{0} \lambda_{0}$ are generated. Using these inter arrival times, $t_{i n_{i}-1}$ is obtained. One inter-arrival time $\left(t_{i n_{i}}-t_{i n_{i}-1}\right)$ having exponential distribution with parameter $\left(1-p_{0}\right) \lambda_{0}$ is generated.

Steps 1 to 4 are repeated for $r=20,25,35,50$. The real life examples that are quoted in Section 2 justifies the consideration of $r$ as 20, 25 (moderate sample) and $r=30,50$ (large samples). In the example of heart disease, the number of patients will be moderately large and among them the cases of cardiac arrests (death due to fatal shock) will be small. To get the non-zero number of cases of failure due to fatal shocks, $r$ needs to be moderately large. The example of credit scoring systems, the number of loan (credit) accounts in any financial company is large and hence, the use of values of $r$ as 35, 50 .

The statistics $n, x_{.}, t$.. and $t^{\prime}$ are computed using which estimators of $S_{1}(t)$ are obtained and for given set of parameters, the survival probability $S_{1}(t)$ is computed for $t=0.5(0.25) 2.0$ units of time. 


\section{Modelling Survival Probability When Threshold $\left(U_{i}\right)$ Is Random Variable}

The threshold of the component or system is considered as random variable by many researchers. It is trivial assumption as the damage sustaining capacity depends on threshold and threshold of the component may vary due to raw materials used in its manufacturing, technology with which it is manufactured, inbuilt capacity, physical properties, random factors that influence its shock sustaining capacity, etc. It is assumed that threshold $U_{i}$ of the component is exponential random variable with parameter $\sigma, \sigma>0$.

The survival probability of the component at time $t$ is given by:

$$
\begin{aligned}
S_{2}(t) & =\sum_{k=0}^{\infty} \frac{\mathrm{e}^{(-p \lambda t)}(p \lambda t)^{k}}{k !} \mathrm{e}^{-(1-p) \lambda t}\left(\frac{\theta}{\theta+\sigma}\right)^{k} \\
& =\mathrm{e}^{-(1-p) \lambda t} \mathrm{e}^{(-p \lambda t)} \sum_{k=0}^{\infty} \frac{\left(\frac{\theta}{\theta+\sigma}\right)^{k}(p \lambda t)^{k}}{k !} \\
& =\mathrm{e}^{-\lambda t\left(1-\frac{p \theta}{\sigma+\theta}\right)}
\end{aligned}
$$

$S_{2}(0)=1, S_{2}(\infty)=0, S_{2}(t) \uparrow t$, implying that the survival probability of the component is non-increasing function of time $t$.

\subsection{Assessment of the Survival Probability}

Suppose $r$ components each with threshold $U_{i}, i=1,2, \cdots, r$ having life distribution $H(t)=1-S_{2}(t)$ are subjected to life testing experiment and the experiment is conducted until all of them fail. Continuing the life testing experiment as in Section 3.

The joint distribution $L_{2}$ of all the involved r.v.'s is given by

$$
L_{2}=p^{n-r_{2}} \lambda^{n} \cdot(1-p)^{r_{2}} \theta^{n \cdot-r} \mathrm{e}^{-p \lambda t} \cdot \mathrm{e}^{-\lambda t^{\prime}} \cdot \mathrm{e}^{-\theta x} \cdot\left(\frac{\sigma}{\sigma+\theta}\right)^{r_{1}} \sigma^{r} \mathrm{e}^{-u}
$$

where,

$$
\begin{gathered}
n_{.}=n_{1}+n_{2}, t^{\prime}=\sum_{i=1}^{r_{2}}\left(t_{n_{i}}-t_{n_{i}-1}\right), x_{. .}=\sum_{i=1}^{r} \sum_{j=1}^{n_{i}-1} x_{i j}, \\
t_{. .}=\sum_{i=1}^{r_{1}} t_{n_{i}}+\sum_{i=1}^{r_{2}} t_{n_{i}-1}-\sum_{i=1}^{r_{2}} t_{n_{i}}, u=\sum_{i=1}^{r} u_{i} .
\end{gathered}
$$

and the maximum likelihood estimators of $p, \lambda, \sigma$ and $\theta$ are obtained as

$$
\left.\begin{array}{l}
\hat{p}=\frac{t^{\prime}\left(n-r_{2}\right)}{t^{\prime} n+t_{. .} r_{2}} \\
\hat{\lambda}=\frac{n t^{\prime}+r_{2} t . .}{t^{\prime}\left(t_{. .}+t^{\prime}\right)} \\
\hat{\sigma}=\frac{\left(r_{1}+r-n\right) \theta+x \theta^{2}}{n-r-x \theta} \\
\hat{\theta}=\frac{\sigma\left(\sigma \sum_{i=1}^{r} u_{i}-r\right)}{r_{1}+r-\sigma \sum_{i=1}^{r} u_{i}}
\end{array}\right\}
$$


where $\hat{\sigma}$ and $\hat{\theta}$ are to be obtained numerically using Newton-Raphson method. MLE $\hat{S}_{2}(t)$ of survival function $S_{2}(t)$ is obtained using the MLEs of parameters in expression (11).

\subsection{Simulation Study}

The estimators are computed using Monte-Carlo simulation for random threshold $U_{i}$ case. For the $I^{\text {th }}$ component, the random variables $U_{i}, t_{i 1}, t_{i 2}, \cdots, t_{i n_{i}}, X_{i 1}, \cdots, X_{i n_{i}-1}$ are generated as follows:

For given value of $\sigma=\sigma_{0}$ an exponential random variable $U_{i}$ is generated and the remaining procedures are same as in Step 1 to Step 4 of Section 4 . On the same lines estimators of $S_{2}(t)$ are computed for same set of $r$ and $t$ values of Section 4.

\section{Comparison of Estimators}

$M=10000$ simulations are carried out to compute mean square errors (MSEs) of $\hat{S}_{i}(t), i=1,2$ using the formula

$$
\operatorname{MSE}_{i}\left(\hat{S}_{i}(t)\right)=\frac{\sum_{j=1}^{M}\left(S_{i}(t)-\hat{S}_{i j}(t)\right)^{2}}{M}, i=1,2
$$

Table 1 and Table 2 provide the relative efficiencies (E) of $\hat{S}_{2}(t)$ as compared to $\hat{S}_{1}(t)$ which is computed as the ratio of $\operatorname{MSE}_{1}\left(\hat{S}_{1}(t)\right)$ to $\operatorname{MSE}_{2}\left(\hat{S}_{2}(t)\right)[17]$.

Table 1. Survival probabilities, MSEs and Efficiencies for $p=0.4, \lambda=0.8, u=1.2$, $\sigma=0.83, \theta=0.6$.

\begin{tabular}{|c|c|c|c|c|c|c|c|c|c|c|}
\hline \multirow{3}{*}{$\begin{array}{c}t \\
0.50\end{array}$} & \multirow{3}{*}{$\begin{array}{r}S_{1}(t) \\
0.73\end{array}$} & \multirow{3}{*}{$\begin{array}{l}S_{2}(t) \\
0.72\end{array}$} & \multirow{2}{*}{\multicolumn{2}{|c|}{$\begin{array}{c}r=20 \\
\text { MSE \& E }\end{array}$}} & \multirow{2}{*}{\multicolumn{2}{|c|}{$\begin{array}{c}r=25 \\
\text { MSE \& E }\end{array}$}} & \multirow{2}{*}{\multicolumn{2}{|c|}{$\begin{array}{c}r=35 \\
\text { MSE \& E }\end{array}$}} & \multirow{2}{*}{\multicolumn{2}{|c|}{$\begin{array}{c}r=50 \\
\text { MSE \& E }\end{array}$}} \\
\hline & & & & & & & & & & \\
\hline & & & $\begin{array}{l}0.0051 \\
0.0037\end{array}$ & 1.37 & $\begin{array}{l}0.0044 \\
\mathbf{0 . 0 0 2 6}\end{array}$ & 1.66 & $\begin{array}{l}0.0037 \\
\mathbf{0 . 0 0 1 7}\end{array}$ & 2.14 & $\begin{array}{l}0.0034 \\
0.0012\end{array}$ & 2.95 \\
\hline 0.75 & 0.62 & 0.61 & $\begin{array}{l}0.0076 \\
0.0058\end{array}$ & 1.32 & $\begin{array}{l}0.0066 \\
\mathbf{0 . 0 0 4 1}\end{array}$ & 1.60 & $\begin{array}{l}0.0056 \\
\mathbf{0 . 0 0 2 7}\end{array}$ & 2.06 & $\begin{array}{l}0.0053 \\
0.0019\end{array}$ & 2.84 \\
\hline 1.00 & 0.53 & 0.51 & $\begin{array}{l}0.0090 \\
0.0071\end{array}$ & 1.27 & $\begin{array}{l}0.0079 \\
\mathbf{0 . 0 0 5 2}\end{array}$ & 1.53 & $\begin{array}{l}0.0068 \\
\mathbf{0 . 0 0 3 4}\end{array}$ & 1.99 & $\begin{array}{l}0.0064 \\
0.0024\end{array}$ & 2.73 \\
\hline 1.25 & 0.45 & 0.43 & $\begin{array}{l}0.0094 \\
0.0078\end{array}$ & 1.21 & $\begin{array}{l}0.0083 \\
0.0057\end{array}$ & 1.47 & $\begin{array}{l}0.0072 \\
\mathbf{0 . 0 0 3 8}\end{array}$ & 1.91 & $\begin{array}{l}0.0069 \\
0.0026\end{array}$ & 2.62 \\
\hline 1.50 & 0.39 & 0.37 & $\begin{array}{l}0.0091 \\
0.0079\end{array}$ & 1.15 & $\begin{array}{l}0.0081 \\
\mathbf{0 . 0 0 5 8}\end{array}$ & 1.41 & $\begin{array}{l}0.0071 \\
0.0039\end{array}$ & 1.83 & $\begin{array}{l}0.0068 \\
0.0027\end{array}$ & 2.51 \\
\hline 1.75 & 0.33 & 0.31 & $\begin{array}{l}0.0083 \\
0.0076\end{array}$ & 1.09 & $\begin{array}{l}0.0075 \\
0.0056\end{array}$ & 1.34 & $\begin{array}{l}0.0066 \\
\mathbf{0 . 0 0 3 8}\end{array}$ & 1.75 & $\begin{array}{l}0.0064 \\
\mathbf{0 . 0 0 2 7}\end{array}$ & 2.40 \\
\hline 2.00 & 0.28 & 0.26 & $\begin{array}{l}0.0074 \\
0.0072\end{array}$ & 1.03 & $\begin{array}{l}0.0067 \\
\mathbf{0 . 0 0 5 2}\end{array}$ & 1.28 & $\begin{array}{l}0.0059 \\
\mathbf{0 . 0 0 3 5}\end{array}$ & 1.68 & $\begin{array}{l}0.0058 \\
0.0025\end{array}$ & 2.30 \\
\hline
\end{tabular}

Figures in bold represents $\operatorname{MSE}_{2}\left(\hat{S}_{2}(t)\right)$. 
Table 2. Survival probabilities, MSEs and Efficiencies for $p=0.6, \lambda=0.4, u=0.9$, $\sigma=1.1, \theta=0.7$.

\begin{tabular}{|c|c|c|c|c|c|c|c|c|c|c|}
\hline \multirow{2}{*}{$t$} & \multirow{2}{*}{$S_{1}(t)$} & \multirow{2}{*}{$S_{2}(t)$} & \multicolumn{2}{|c|}{$r=20$} & \multicolumn{2}{|c|}{$r=25$} & \multicolumn{2}{|c|}{$r=35$} & \multicolumn{2}{|c|}{$r=50$} \\
\hline & & & MSE & & MSE & & MSE & & MSE & \\
\hline 0.50 & 0.87 & 0.86 & $\begin{array}{l}0.0027 \\
\mathbf{0 . 0 0 1 7}\end{array}$ & 1.55 & $\begin{array}{l}0.0021 \\
\mathbf{0 . 0 0 1 0}\end{array}$ & 2.07 & $\begin{array}{l}0.0019 \\
0.0005\end{array}$ & 3.51 & $\begin{array}{l}0.0018 \\
\mathbf{0 . 0 0 0 4}\end{array}$ & 4.84 \\
\hline 0.75 & 0.81 & 0.79 & $\begin{array}{l}0.0048 \\
\mathbf{0 . 0 0 3 2}\end{array}$ & 1.50 & $\begin{array}{l}0.0039 \\
\mathbf{0 . 0 0 1 9}\end{array}$ & 2.02 & $\begin{array}{l}0.0035 \\
0.0010\end{array}$ & 3.42 & $\begin{array}{l}0.0035 \\
\mathbf{0 . 0 0 0 7}\end{array}$ & 4.71 \\
\hline 1.00 & 0.75 & 0.74 & $\begin{array}{l}0.0068 \\
\mathbf{0 . 0 0 4 7}\end{array}$ & 1.44 & $\begin{array}{l}0.0057 \\
\mathbf{0 . 0 0 2 9}\end{array}$ & 1.98 & $\begin{array}{l}0.0053 \\
0.0016\end{array}$ & 3.34 & $\begin{array}{l}0.0052 \\
0.0011\end{array}$ & 4.57 \\
\hline 1.25 & 0.70 & 0.68 & $\begin{array}{l}0.0087 \\
\mathbf{0 . 0 0 6 3}\end{array}$ & 1.38 & $\begin{array}{l}0.0073 \\
\mathbf{0 . 0 0 3 8}\end{array}$ & 1.93 & $\begin{array}{l}0.0068 \\
0.0021\end{array}$ & 3.25 & $\begin{array}{l}0.0068 \\
0.0015\end{array}$ & 4.44 \\
\hline 1.50 & 0.65 & 0.63 & $\begin{array}{l}0.0102 \\
\mathbf{0 . 0 0 7 7}\end{array}$ & 1.32 & $\begin{array}{l}0.0088 \\
0.0047\end{array}$ & 1.88 & $\begin{array}{l}0.0082 \\
0.0026\end{array}$ & 3.17 & $\begin{array}{l}0.0082 \\
0.0019\end{array}$ & 4.32 \\
\hline 1.75 & 0.60 & 0.58 & $\begin{array}{l}0.0113 \\
0.0090\end{array}$ & 1.26 & $\begin{array}{l}0.0099 \\
\mathbf{0 . 0 0 5 4}\end{array}$ & 1.82 & $\begin{array}{l}0.0094 \\
0.0030\end{array}$ & 3.09 & $\begin{array}{l}0.0093 \\
0.0022\end{array}$ & 4.19 \\
\hline 2.00 & 0.56 & 0.54 & $\begin{array}{l}0.0121 \\
0.0101\end{array}$ & 1.21 & $\begin{array}{l}0.0107 \\
\mathbf{0 . 0 0 6 1}\end{array}$ & 1.77 & $\begin{array}{l}0.0102 \\
0.0034\end{array}$ & 3.01 & $\begin{array}{l}0.0102 \\
0.0025\end{array}$ & 4.07 \\
\hline
\end{tabular}

Figures in bold represent $\operatorname{MSE}_{2}\left(\hat{S}_{2}(t)\right)$.

\section{Results and Conclusion}

From the tables it is clear that survival functions $S_{1}(t)$ and $S_{2}(t)$ decrease as $t$ increases. MLEs of parameters maximize the likelihood of the joint distribution function. Further, using invariance property of MLE, it is easy to obtain the MLE of survival function. In case of random threshold, the MLEs of parameters are obtained numerically and hence asymptotic distribution of MLEs cannot be obtained which is a limitation of this study. MLE, $\hat{S}_{2}(t)$ overestimates the true value of survival function $S_{2}(t)$ and $\hat{S}_{1}(t)$ underestimates the true value of survival function $S_{1}(t)$, the reason for which may be simulated random variables in case of simulation study/a chance error if we are considering the real data. As the value of $r$ increases, MSEs decrease. $\hat{S}_{2}(t)$ performs better than $\hat{S}_{1}(t)$, so the study advocates consideration of threshold as a random variable and is a realistic assumption also.

\section{Acknowledgements}

The second author is thankful to Department of Science and Technology-Innovation in Science Pursuit for Inspired Research (DST-INSPIRE) for financial support.

\section{Conflicts of Interest}

The authors declare no conflicts of interest regarding the publication of this paper. 


\section{References}

[1] Esary, J.D., Marshall, A.W. and Proschan, F. (1973) Shock Models and Wear Processes. The Annals of Probability, 1, 627-649. https://doi.org/10.1214/aop/1176996891

[2] Abdel-Hameed, M.S. and Proschan, F. (1973) Nonstationary Shock Models. Stochastic Processes and their Applications, 1, 383-404. https://doi.org/10.1016/0304-4149(73)90019-7

[3] Barlow, R.E. and Proschan, F. (1975) Statistical Theory of Reliability and Life Testing. Holt. Rinhart and Winston, Inc., New York.

[4] Abdel-Hameed, M.S. and Proschan, F. (1975) Shock Models with Underlying Birth Processes. Journal of Applied Probability, 12, 18-28. https://doi.org/10.2307/3212403

[5] Klefsjo, B. (1981) Survival under the Pure Birth Shock Model. Journal of Applied Probability, 18, 554-560. https://doi.org/10.2307/3213305

[6] Ross, S.M. (1981) Generalised Poisson Shock Models. The Annals of Probability, 9 , 896-898. https://doi.org/10.1214/aop/1176994318

[7] Posner, M.J.M. and Zuckerman, D. (1986) Semi-Markov Shock Models with Additive Damage. Advances in Applied Probability, 18, 772-790. https://doi.org/10.2307/1427187

[8] Anderson, K.K. (1987) Limit Theorems for General Shock Models with Infinite Mean Inter Shock Times. Journal of Applied Probability, 24, 449-456. https://doi.org/10.2307/3214268

[9] Savits, T.H. (1988) Some Multivariate Distributions Derived from a Non-Fatal Shock Model. Journal of Applied Probability, 25, 383-390. https://doi.org/10.2307/3214446

[10] Gut, A. (1990) Cumulative Shock Models. Advances in Applied Probability, 22, 504-507. https://doi.org/10.2307/1427554

[11] Skoulakis, G. (2000) A General Shock Model for a Reliability System. Journal of Applied Probability, 37, 925-935. https://doi.org/10.1239/jap/1014843073

[12] Mallor, F. and Santos, J. (2003) Classification of Shock Models in System Reliability. Monografias del Seminario Matematico García de Galdeano, 27, 405-412.

[13] Munoli, S.B. and Bhat, S.V. (2011) Reliability Estimation in Shock Model When Successive Shocks Cause Greater Damage (Non-Accumulating Damages). Research Journal of Mathematics and Statistics, 3, 61-66. https://doi.org/10.1155/2011/721962

[14] Lindskog, F., and McNeil, A.J. (2003) Common Poisson Shock Models: Applications to Insurance and Credit Risk Modeling. ASTIN Bulletin: The Journal of the $I A A, 33,209-238$. https://doi.org/10.1017/S0515036100013441

[15] Stepanova, M. and Thomas, L. (2002) Survival Analysis Methods for Personal Loan Data. Operations Research, 50, 277-289. https://doi.org/10.1287/opre.50.2.277.426

[16] Deshpande, J.V. and Purohit, S.G. (2005) Life Time Data: Statistical Models and Methods. World Scientific Publishing Company, Singapore.

https://doi.org/10.1142/5988

[17] Kale, B.K. (1999) A First Course on Parametric Inference. Narosa Publishing House, New Delhi. 